\title{
Revascularização miocárdica de urgência após complicação de angioplastia transluminal coronária: abordagem cirúrgica atual
}

Luís Alberto DALLAN*, Sérgio de Almeida OLIVEIRA*, Hedy CECCHY^, Siguemituso ARIÊ*, Alexandre SABINO NETO*, José Carlos R. IGLÉZIAS*, Geraldo VERGINELLI*, Adib D. JATENE*

DALLAN, L. A.; OLIVEIRA, S. A.; CECCHY, H.; AIRÊ, S.; SABINO NETO, A.; IGLÉZIAS, J. C. R.; VERGINELLI, G.; JATENE, A. D. - Revascularização miocárdica de urgência após complicação de angioplastia transluminal coronária: abordagem cirúrgica atual. Rev. Bras. Cir. Cardiovasc., 5(1): 42-53, 1990.

RESUMO: Entre julho de 1981 e fevereiro de 1990, foram realizados no INCOR 3016 angioplastias transluminais coronárias (ATC), em 2431 pacientes portadores de insuficiência coronária em suas diferentes manifestaçōes. A cirurgia de revascularização do miocárdio de urgência foi necessária em $79(3,2 \%)$ desses pacientes, dos quais $32(40,5 \%)$ infartaram e $12(15,2 \%)$ faleceram. Dos 1530 pacientes submetidos eletivamente a ATC por angina estável, 11,9\% (7/59) faleceram no intra ou no pós-operatório. Dentre os 112 pacientes em que a ATC foi realizada de urgência devido a angina instável, 18,2\% (2/11) evoluíram para óbito operatório. Nos 789 já infartados previamente à ATC, a mortalidade cirúrgica foi de $33,3 \%(3 / 9)$. A análise estatística demonstrou mortalidade mais elevada em pacientes idosos, em pacientes com dissecção do tronco da artéria coronária esquerda ou trombose coronária tardia e, especialmente, naqueles com instabilidade hemodinâmica após a ATC. O tempo decorrido entre a complicação da ATC e a revascularização do miocárdio também constitui fator agravante no prognóstico. O número de cirurgias de urgência após ATC diminuiu significativamente nos últimos dois anos (10/1351), a despeito do grau progressivo de complexidade das lesóes dilatadas. 0 emprego de balōes com extremidade atraumática e, especialmente, de cateter de reperfusāo (Stack), que mantém o fluxo coronário mesmo diante do desprendimento da placa ateromatosa, contribuiu, decisivamente, para esses resultados. As modificaçōes introduzidas na sistematização operatória, principalmente o uso de cardioplegia sangüínea com indução normotérmica e administrada por vias anterógrada e retrógrada, também propiciaram melhores resultados cirúrgicos. A associação desses procedimentos vem se mostrando benéfica, não tendo sido observados óbitos nos últimos 14 meses.

DESCRITORES: miocárdio, revascularização, angioplastia; miocárdio, revascularização, cirurgia; proteção miocárdica, cardioplegia.

\section{INTRODUÇÃO}

A angioplastia transluminal coronária (ATC) vem sendo cada vez mais empregada no tratamento de pacientes selecionados, portadores de doença arterial co- ronária obstrutiva. Entretanto, apesar dos avanços da técnica e aprimoramento dos equipamentos utilizados, o procedimento não é isento de riscos. A oclusão da artéria dilatada ocorre entre 2 e $12,2 \%$ na experiência de diferentes Serviços ${ }^{5}, 11,17,22,23$. Os mecanismos de

Trabalho realizado no Instituto do Coração do Hospital das Clínicas da Faculdade de Medicina da Universidade de São Paulo. Săo Paulo, SP, Brasil

Apresentado ao 17: Congresso Nacional de Cirurgia Cardiaca. Belo Horizonte, MG, 6 e 7 de abril, 1990.

- Do Instituto do Coração do Hospital das Clínicas da Faculdade de Medicina da Universidade de São Paulo.

Endereço para separatas: Luis Alberto Dallan. Av. Dr. Enéas Carvalho de Aguiar, 44. Divisão Cirúrgica. 05403 Săo Paulo, SP, Brasil. 
DALlan, L. A.; OliveiRA, S. A.; CECCHY, H.; AIRÊ, S.; SABINO NETO, A.; IGLÉZIAS, J. C. R.; VERGINELLI, G.; JATENE, A. D. - Revascularização miocárdica de urgência após complicação de angioplastia transluminal coronária: abordagem cirúrgica atual. Rev. Bras. Cir. Cardiovasc., 5(1): 42-53, 1990.

oclusāo arterial incluem a hemorragia subintimal, a dissecção da camada íntima da artéria junto à placa aterosclerótica, o espasmo coronário, a trombose local, ou a associação destes eventos ${ }^{7}, 35$. A oclusão aguda da artéria coronária freqüentemente acarreta isquemia de extensas áreas miocárdicas, podendo provocar o infarto, se rápidas medidas protetoras não forem tomadas. A revascularização cirúrgica de urgência é uma das intervenções utilizadas para minimizar os efeitos deletérios da isquemia. Entretanto, algumas vezes, apesar da pronta atuação da equipe cirúrgica, o comprometimento miocárdico que se instala nesse período pode acarretar graves seqüelas, aumentando a morbidade e a mortalidade $^{8}$. Por outro lado, alguns trabalhos recentes sugerem que modificaçōes da técnica de reperfusão cirúrgica ${ }^{29}$. ${ }^{30},{ }^{32}$ podem aumentar as possibilidades de recuperação do miocárdio isquêmico.

O uso de cateteres que permitem a manutenção do fluxo coronário, até que se promova a revascularizaçăo cirúrgica, e o emprego de proteção miocárdica, através de solução cardioplégica por via anterógrada e retrógrada, têm contribuído para minimizar esses problemas.

Este estudo descreve a experiência com 79 pacientes consecutivos do Instituto do Coração, submetidos a cirurgia de urgência por complicação de ATC.

\section{CASUÍSTICAS E MÉTODOS}

No período de julho de 1981 a fevereiro de 1990 , foram realizadas 3016 ATC em 2431 pacientes no Instituto do Coração. Esse número compreende tanto as angioplastias eletivas, quanto aquelas realizadas em pacientes com angina incontrolável ou infarto agudo do miocárdio instalado (IAM). Desse total, $79(3,2 \%)$ pacientes necessitaram revascularização do miocárdio em caráter de urgência, devido a complicação no procedimento. Dos 1530 pacientes portadores de insuficiência coronária crônica submetidos a ATC, 59 (3,8\%) foram operados de urgência. Nove $(1,1 \%)$ dentre 789 pacientes do grupo de IAM e $11(9,8 \%)$ dentre 112 pacientes com quadro de síndrome intermediária (SI) foram igualmente revascularizados cirurgicamente de urgência.

O sexo masculino predominou em $56(70,9 \%)$ pacientes e a faixa etária variou de 28 a 80 anos, com média de $56,8 \pm 8$ anos. Setenta e cinco $(94,9 \%)$ eram de cor branca, $2(2,5 \%)$ de cor negra e $2(2,5 \%)$ amarela.

A indicação da operação deu-se por deslocamento de placa ateromatosa no local da ATC em $59(74,7 \%)$ pacientes, por dissecção do tronco da coronária esquerda (TCE) em $8(10,1 \%)$, por trombose tardia no local dilatado em $7(8,9 \%)$, por reoclusão repetitiva da artéria dilatada em $4(5,1 \%)$ e $1(1,3 \%)$ paciente foi à cirurgia devido a angina incontrolável pós ATC, apesar da artéria estar pérvia.

Em 46 (52,8\%) pacientes, havia lesão de apenas uma artéria coronária ( $\geqslant 70 \%$ ). Havia comprometimento biarterial em $22(27,8 \%)$ pacientes, e em três ou mais artérias em $16(13,9 \%)$.

A artéria em que mais freqüentemente ocorreu complicação da ATC foi a descendente anterior (DA), compreendendo $57(72,1 \%)$ pacientes. Seguiu-se a artéria coronária direita (CD) em $18(22,8 \%)$ pacientes e a artéria circunflexa $(C X)$ ou seus ramos em $4(5,1 \%)$ pacientes.

Trinta e um $(39,2 \%)$ pacientes foram revascularizados em período inferior a duas horas após a complicação da ATC. Em 25 (35,4\%) pacientes esse intervalo situou-se entre duas e quatro horas; em $6(7,6 \%)$ entre quatro e seis horas; em dois $(2,5 \%)$ pacientes esse periodo foi superior a seis horas e em $15(18,9 \%)$ não foi possível determiná-lo com precisão. É importante destacar que toda ACT eletiva é precedida de completo preparo do paciente, no que tange a exames pré-operatórios, tricotomia, tipagem sangüínea, além de sala operatória e equipe cirúrgica à disposição para eventual emergência.

Vinte e dois $(27,9 \%)$ pacientes apresentaram comprometimento severo na função ventricular esquerda após a complicação da ATC. Destes, nove foram à cirurgia sob massagem cardiaca externa. Os 57 (72,1\%) restantes encontravam-se em situação hemodinâmica estável. Recentemente, tem-se logrado manter o paciente em razoáveis condiçōes hemodinâmicas, mesmo diante da oclusão total da artéria coronária. Isto tem sido possível pelo emprego de cateteres de reperfusão ${ }^{15}$ que permitem restabelecer o fluxo através da artéria obstruída até que se promova a revascularização cirúrgica.

Em $71(89,9 \%)$ pacientes, empregaram-se apenas enxertos de veia safena na revascularização do miocárdio. Nos $8(10,21 \%)$ pacientes restantes, utilizou-se enxerto com artéria mamária isolada ou associada com pontes de veia safena. A média de enxertos por paciente foi de 1,57 .

A proteção miocárdica durante a revascularizção do miocárdio foi variável em nossa casuística. Em 36 $(45,6 \%)$ pacientes empregou-se o pinçamento aórtico intermitente durante a realização das anastomoses. Em 36 outros pacientes utilizou-se cardioplegia acelular (Saint Thomas), e em $2(2,5 \%)$ a revascularização foi realizada sem o auxílio da circulação extracorpórea (CEC). Nos últimos 5 (6,4\%) pacientes empregou-se a técnica preconizada por PARTINGTON et alii ${ }^{27}$, de cardioplegia com indução normotérmica $\left(36^{\circ} \mathrm{C}\right)$. 
DALLAN, L. A.; OliVEIRA, S. A.; CECCHY, H.; AIRÊ, S.; SABINO NETO, A.; IGLÉZIAS, J. C. R.; VERGINELLI, G.; JATENE, A. D. - Revascularização miocárdica de urgência após complicação de angioplastia transluminal coronária: abordagem cirúrgica atual. Rev. Bras. Cir. Cardiovasc., 5(1): 42-53, 1990.

Nessa técnica, a infusão de solução cardioplégica é realizada por via anterógrada (raiz da aorta) e retrógrada (seio coronário) sob baixa pressão e associada à descompressão das câmaras cardíacas esquerdas, sendo seu veículo de infusão o próprio sangue, na proporção 1:4. A solução inicial contém 20 a $25 \mathrm{mEq} /$ de cloreto de potássio, $13 \mathrm{mM} / /$ de aspartato e de glumatato de sódio, 0,15 a $0,25 \mathrm{mM} / \mathrm{l}$ de adenina citrato dextrose (ACD), complementada com bicarbonato de sódio para se obter um pH 7,7-7,8. Contém também glicose a $50 \%$, visando elevar a osmolaridade para níveis de 380 a $400 \mathrm{~m}$ Osm. O fluxo de infusão inicial é de 250 a $350 \mathrm{ml} / \mathrm{min}$, até a total parada dos batimentos cardía$\cos$, quando é reduzido a $150 \mathrm{ml} / \mathrm{min}$, até completarem-se cinco minutos. Essa solução inicial é mantida em torno de $36^{\circ} \mathrm{C}$. Em seguida, é substituída por solução gelada $\left( \pm 4^{\circ} \mathrm{C}\right)$ e com menor concentração de potássio (8-10 mEq/), repetida, se necessário, a cada 20 minutos. Após a realização da(s) ponte(s), o coração é reperfundido por três minutos com esta última solução cardioplégica, em normotermia, com fluxo de $150 \mathrm{ml} / \mathrm{min}$ e pressão de infusão não superior a $50 \mathrm{mmHg}$, após o que a pinça de aorta é removida e restabelecido o fluxo sangüineo coronário. Nessa fase, deve-se manter as câmaras cardiacas esquerdas descomprimidas e o paciente deve ser mantido em circulação assistida até a completa recuperação dos batimentos cardíacos e estabilização dos parâmetros hemodinâmicos.

\section{RESULTADOS}

Setenta e nove $(3,2 \%)$, dentre os 2431 pacientes consecutivos submetidos a 3016 ATC no Instituto do Coração, necessitaram cirurgia de urgência. Doze $(15,2 \%)$ deles vieram a falecer no período hospitalar. Houve $7(11,9 \%)$ óbitos entre os 59 pacientes do grupo portador de insuficiência coronária crônica, 3 (33,3\%) óbitos entre os nove que haviam sofrido infarto agudo do miocárdio, e dois $(18,2 \%)$ óbitos entre os 11 pacientes com síndrome intermediária (Tabela 1).

A distribuiçăo anual das ATC com as respectivas cirurgias de urgência e mortalidade pode ser observada na Tabela 2. Pode-se verificar que, apesar do número crescente de procedimentos, houve uma diminuição acentuada no número de cirurgias de urgência nos últimos dois anos. Foram operados $8(1,2 \%)$ pacientes em 1988 , dos quais $2(25 \%)$ faleceram; e $2(0,3 \%)$ de janeiro de 1989 a fevereiro de 1990 , tendo ambos sobrevivido.

Quatro $(6,8 \%)$, dentre os 59 pacientes cuja complicaçāo da ATC foi o descolamento de placa ateromatosa no local dilatado, vieram a falecer. Dos oito pacientes com dissecção do tronco da coronária esquerda, 4 (50\%) foram a óbito, Dentre os sete que apresentaram trombose arterial tardia, $3(42,9 \%)$ faleceram. Um $(25 \%)$ dos quatro pacientes com reoclusão repetitiva arterial fale- ceu, enquanto aquele operado por angina incontrolável sobreviveu. $O$ estudo estatístico $\left(X^{2}\right)$ revelou diferença significante $(P=0,001)$ entre a proporçăo de óbitos nos três primeiros grupos (Tabela 3 ).

TABELA 1

RELAÇAOO DAS ATC REALIZADAS EM DIFERENTES CONDICOOES CLINICAS COM AS RESPECTIVAS OPERAÇÓES DE URGENCIA E MORTALIDADE

\begin{tabular}{lcrr}
\hline Condiçáo clínica & $\begin{array}{c}\text { N: de } \\
\text { pacientes }\end{array}$ & $\begin{array}{c}\text { Operaçōes de } \\
\text { urgéncia }\end{array}$ & Mortalidade \\
\hline $\begin{array}{l}\text { Insuficiência } \\
\text { coronária crónica }\end{array}$ & 1530 & $59(3,8 \%)$ & $7(11,9 \%)$ \\
$\begin{array}{l}\text { Infarto agudo do } \\
\text { miocárdio }\end{array}$ & 789 & $9(1,1 \%)$ & $3(33,3 \%)$ \\
$\begin{array}{l}\text { Síndrome intermediária } \\
\text { Total de pacientes }\end{array}$ & 112 & $11(9,8 \%)$ & $2(18,2 \%)$ \\
\hline Total de Procedimentos $=3016$ & $79(3,2 \%)$ & $12(15,2 \%)$ \\
\hline
\end{tabular}

TABELA 2

RELAÇÃO ANUAL ENTRE O NÚMERO DE ANGIOPLASTIAS E CIRURGIAS DE URGENCIA, COM AS RESPECTIVAS MORTALIDADES

\begin{tabular}{lcrc}
\hline Ano & $\begin{array}{c}N^{\circ} \\
\text { angioplastias }\end{array}$ & $\begin{array}{c}\text { Cirurg. } \\
\text { urgéncia }\end{array}$ & Mortalidade \\
\hline 1981 & 3 & - & - \\
1982 & 70 & $4(5,7 \%)$ & - \\
1983 & 151 & $11(7,3 \%)$ & $1(9,1 \%)$ \\
1984 & 188 & $12(6,4 \%)$ & - \\
1985 & 350 & $9(2,6 \%)$ & $1(11,1 \%)$ \\
1986 & 233 & $20(8,6 \%)$ & $3(15,0 \%)$ \\
1987 & 660 & $13(1,9 \%)$ & $5(38,4 \%)$ \\
1988 & 643 & $8(1,2 \%)$ & $2(25,0 \%)$ \\
$1989 / \mathrm{fev} .90$ & 708 & $2(0,3 \%)$ & - \\
\hline Total & 3016 & $79(2,6 \%)$ & $12(15,2 \%)$ \\
\hline
\end{tabular}

TABELA 3

COMPLICAÇÓES DA ATC QUE LEVARAM À CIRURGIA DE URGENCIA

Indicação

N. pacientes Mortalidade

Desprendimento de placa no local

da ATC

Dissecção de TCE

Trombose tardia

Reoclusăo repetitiva

Angina incontrolável 
DALLAN, L. A.; OlIVEIRA, S. A.; CECCHY, H.; AIRÊ, S.; SABINO NETO, A.; IGLÉZIAS, J. C. R.; VERGINELLI, G.; JATENE, A. D. - Revascularizaçăo miocárdica de urgência após complicaçăo de angioplastia transluminal coronária: abordagem cirúrgica atual. Rev. Bras. Cir. Cardiovasc., 5(1): 42-53, 1990.

O maior número de óbitos concentrou-se na faixa etária entre os 50 e 70 anos. Dos 18 pacientes com idade entre 40 e 49 anos, observamos $1(5,5 \%)$ óbito. Entre os 29 pacientes em faixa etária 50-59 anos, 4 $(13,8 \%)$ faleceram. Na década $60-69$ anos, incluíram-se 20 pacientes, com 5 ( $25 \%$ ) óbitos, e, dos cinco pacientes com idade variável de 70 a 79 anos, $2(40 \%)$ faleceram. Nas outras faixas etárias, não se observou mortalidade.

Dentre 46 pacientes com comprometimento uniarterial coronário prévio ( $\geqslant 70 \%)$, observamos $7(15,2 \%)$ óbitos. Dos 22 pacientes com comprometimento de duas artérias coronárias, $3(13,6 \%)$ faleceram, e, dos 11 restantes, com três ou mais artérias lesadas, houve 2 $(18,2 \%)$ b́bitos. O estudo estatístico $\left(X^{2}\right)$ demonstrou não existir diferença significante $(P=0,943)$ entre a proporção de óbito nos três grupos.

Dos 57 pacientes em que a complicação da ATC ocorreu na artéria descendente anterior (DA), 9 (15,8\%) faleceram. Apenas $1(5,5 \%)$ dos 18 pacientes em que a artéria envolvida foi a coronária direita (CD), foi a óbito e $2(50 \%)$, dos quatro pacientes cuja ATC foi para a artéria circunflexa (Cx), faleceram. Apesar da grande variaçăo na porcentagem de óbitos dos três grupos, o teste estatístico $\left(X^{2}\right)$ não apresentou resultado significante no seu confronto $(P=0,079)$.

Em 64 pacientes, foi possivel determinar-se o período decorrido entre a complicação da ATC e a efetiva revascularização do miocárdio. Dos 31 pacientes em que esse intervalo foi igual ou inferior a duas horas, $4(12,9 \%)$ faleceram. Dentre 25 cujo período situou-se entre duas e quatro horas, $4(16 \%)$ foram a óbito. No intervalo quatro a seis horas, enquadraram-se seis pacientes, com 2 (33,3\%) óbitos e 1 (50\%) dos dois pacientes revascularizados acima de seis horas após a complicação da ATC faleceu. Verificou-se variação estatística significante ( $P=0,046)$ na comparação dos óbitos ocorridos nos diferentes períodos (Tabela 4).

A mortalidade foi bastante mais elevada nos pacientes com instabilidade hemodinâmica. Dos 13 pacientes em baixo débito cardíaco após a ATC, $4(30,8 \%)$ faleceram. De outros nove que necessitaram massagem cardíaca externa, $5(55,6 \%)$ não se recuperaram com a revascularização do miocárdio e apenas $3(5,2 \%)$ dos 57 pacientes em condiçōes hemodinâmicas estáveis faleceram. A comparação estatística $\left(X^{2}\right)$ mostrou que existe diferença altamente significante $(P<0,0001)$ na proporção de óbitos entre os três grupos (Tabela 5).

Dos 71 pacientes que receberam apenas enxertos com veia safena, $9(12,6 \%)$ foram a b́bito. Dos oito que receberam enxerto com artéria mamária, $3(37,5 \%)$ faleceram. Não se observou diferença estatística na proporção de óbitos dos pacientes submetidos a enxerto com artéria mamária $(P=0,064)$.
TABELA 4

PERIODO DECORRENTE ENTRE A COMPLICAÇÃODA ATC E A REVASCULARIZAÇÄO DO MIOCÁRDIO

\begin{tabular}{lcc}
\hline$\Delta T$ & N. pacientes & Mortalidade \\
\hline$\leqslant 2$ horas & 31 & $4(12,9 \%)$ \\
$2-4$ horas & 25 & $4(16,0 \%)$ \\
$4-6$ horas & 6 & $2(33,3 \%)$ \\
$>6$ horas & 2 & $1(50,0 \%)$ \\
não determinado & 15 & $1(6,7 \%)$ \\
\hline & & $P=0,046$ \\
\hline
\end{tabular}

TABELA 5

RELAÇĀO ENTRE A CONDIÇĀO HEMODINAMICA PRÉ-OPERATORIA DOS PACIENTES E A MORTALIDADE

\begin{tabular}{lcc}
\hline Condiçáo & N. pacientes & Mortalidade \\
\hline Estável & $57(72,2 \%)$ & $3(5,2 \%)$ \\
Baixo débito cardíaco & $13(16,4 \%)$ & $4(30,8 \%)$ \\
Parada cardiaca & $9(11,3 \%)$ & $5(55,6 \%)$ \\
(sob massagem externa) & 79 & $12(15,2 \%)$ \\
\hline Total & & $P<0,0001$ \\
\hline
\end{tabular}

Entretanto, esse valor situou-se pouco acima do limite preconizado ( $P=0,05)$, somente não sendo significante devido ao pequeno número de pacientes incluídos nesse grupo.

Dos 36 pacientes cuja proteção miocárdica foi cardioplegia acelular, $4(11,1 \%)$ faleceram. Igual número de pacientes foi operado com pinçamento aórtico intermitente; sete $(19,4 \%)$ foram a óbito. Dos cinco pacientes que receberam cardioplegia, não se verificou mortalidade e $1(50 \%)$ dos dois pacientes operados sem CEC faleceu. $O$ teste $X^{2}$ não demonstrou diferença significante $(P=0,384)$ na proporção de óbitos, devido ao pequeno número de pacientes nos dois últimos grupos.

Dentre as complicaçōes mais freqũentes da ATC mal sucedida, destaca-se o IAM. Trinta e dois $(40,5 \%)$ dos 79 pacientes operados de urgência após ATC apresentaram alteração do ECG e/ou elevação significativa de CKMB (>40 U.I.). Dos 59 pacientes pertencentes ao grupo eletivo, $21(35,6 \%)$ infartaram. Seis $(66,6 \%)$ dos nove pacientes que já chegaram ao hospital com IAM, persistiram com o quadro no pós-operatório. Nos três pacientes restantes desse grupo, a trombólise enzimática, associada à revascularizaçāo cirúrgica, possibilitou reverter processo inicial; $5(45,5 \%)$ dos 11 pacientes do grupo S.I. evoluiram para IAM. 
DALlAN, L. A.; OLIVEIRA, S. A.; CECCHY, H.; AIRÊ, S.; SABINO NETO, A.; IGLÉZIAS, J. C. R.; VERGINELLI, G.; JATENE,

A. D. - Revascularização miocárdica de urgência após complicação de angioplastia transluminal coronária: abordagem cirúrgica atual. Rev. Bras. Cir. Cardiovasc., 5(1): 42-53, 1990.

Dentre as demais complicações pós-operatórias, destacamos a insuficiência respiratória em $7(8,9 \%)$ pacientes, insuficiência renal em $6(7,6 \%)$, complicaçōes abdominais (distenção persistente, pancreatite e isquemia mesentérica) em $5(6,3 \%)$, complicaçōes neurológicas (acidente vascular cerebral transitório e/ou confusão mental reversível) em $3(3,8 \%)$, mediastinite necessitando reintervenção cirúrgica em $2(2,5 \%)$. Dois $(2,5 \%)$ pacientes foram submetidos a revisão de hemostasia e $2(2,5 \%)$ apresentaram bloqueio atrioventricular total (BAVT) transitório, necessitando marcapasso temporário.

Dos $12(15,2 \%)$ óbitos observados, $8(66,7 \%)$ tiveram como causa direta a falência miocárdica. Os 4 $(33,3 \%)$ restantes foram conseqüentes a infecção (mediastinite) em $2(16,7 \%)$ pacientes, insuficiência respiratória em $1(8,3 \%)$ e trombose mesentérica em $1(8,3 \%)$ (Tabela 6).

O seguimento tardio, até a presente data, foi possível em $54(80,6 \%)$ dos pacientes sobreviventes. Destes, $45(83,3 \%)$ encontram-se assintomáticos. Cinco $(9,3 \%)$ pacientes apresentam limitaçōes em suas atividades, estando três em classe funcional (C.F.) II e dois em C. F. III. Três $(5,6 \%)$ pacientes foram reoperados, todos devido a evolução da doença coronária. Um deles havia recebido anastomose da artéria mamária interna esquerda para a artéria DA após a ATC e desenvolveu lesão de tronco de coronária esquerda (TCE), sendo acrescentada ponte de veia safena para artéria marginal esquerda (ME). Os demais desenvolveram lesōes obstrutivas em outras artérias do sistema coronário. Foi realizada nova angioplastia em um paciente, por duas vezes, sendo a primeira por lesão na ponte de veia safena para $C D$ (passando de $90 \%$ para $20 \%$ de obstrução) e a outra na própria artéria $\mathrm{CD}$ distal à ponte de safena (passando de $80 \%$ para irregularidades).

Quatro $(7,4 \%)$ pacientes faleceram nesse período. Dois deles tiveram morte súbita, respectivamente, no primeiro e no terceiro ano de pós-operatório. Os outros dois faleceram por sepsis, um deles decorridos três anos da ATC, no pós-operatório de gastrectomia, e o outro após quatro anos, no pós-operatório de revascularização do miocárdio (Tabela 7).

O reestudo hemodinâmico realizado em 23 enxertos de veia safena mostrou $21(91,3 \%)$ pérvios, $1(4,3 \%)$ ocluído e $1(4,3 \%)$ com lesão de $90 \%$ em seu terço médio (submetido a angioplastia). Os três enxertos com artéria mamária estudados encontravam-se pérvios e funcionantes (Tabela 8 ).

\section{DISCUSSĀO}

A ATC é um método alternativo para o tratamento da doença obstrutiva das artérias coronárias ${ }^{6}$. Iniciado por GRUENTZIG et alii ${ }^{14}$, em 1977, passou a ser mais difundido no início dos anos 80. Estima-se, que, em 1990, serāo realizadas aproximadamente 400.000 ATC somente nos EUA ${ }^{38}$.

TABELA 6

PRINCIPAIS COMPLICAÇÓES INTRA E

POSS-OPERATÓRIAS, COM AS RESPECTIVAS CAUSAS DE OBITO

\begin{tabular}{lr}
\hline Complicaçōes & N: pacientes \\
\hline IAM per-operatório & $32(40,5 \%)$ \\
Insuficiência respiratória & $7(8,9 \%)$ \\
Insuficiência renal & $6(7,6 \%)$ \\
Complicaçōes abdominais & $5(6,3 \%)$ \\
Complicaçōes neurológicas & $3(3,8 \%)$ \\
Mediastinite & $2(2,5 \%)$ \\
Reoperação por sangramento & $2(2,5 \%)$ \\
BAVT transitório & $2(2,5 \%)$ \\
\hline
\end{tabular}

CAUSAS DE OBITO $(\mathrm{N}=12)$

Miocárdicas - $8(66,7 \%)$

Não miocárdicas - 4 (33,3\%)

Infecção - $2(16,7 \%)$

Insuficiência respiratória $-1(8,3 \%)$

Trombose mesentérica $-1(8,3 \%)$

TABELA 7

EVOLUÇÃO TARDIA DOS PACIENTES

PACIENTES OPERADOS $=79$

PACIENTES SEGUIDOS $=54(68,4 \%)$

$\mid \begin{aligned} & \text { ASSINTOMÁTICOS }=45(83,3 \%) \\ & \text { SINTOMÁTICOS }=\mid \begin{array}{l}\text { C.F. } \|=3(5,6 \%) \\ \text { C.F. } I I I=2(3,7 \%)\end{array}\end{aligned}$

\begin{tabular}{l|l|l} 
OBITOS & $(7,4 \%)$
\end{tabular} \mid \begin{tabular}{l|l} 
MORTE SÚBITA $=2$ & $\begin{array}{l}1 \text { : ano P.O. } \\
\text { 3: ano P.O. }\end{array}$ \\
SEPSIS $=2$ & $\begin{array}{l}\text { 3: ano - pós gastrectomia } \\
\text { 4: ano - pós revasc. miocárdio }\end{array}$
\end{tabular}

REOPERAÇŌES $=3$ (EVOLUÇĀO DOENÇA CORONÁRIA)

REANGIOPLASTIA $=\mid$ na PS-CD $(90 \rightarrow 20 \%)$

1 pac. por $2 x \quad$ na CD distal à PS $(80 \% \rightarrow$ irreg.)

TABELA 8

REESTUDO HEMODINAMICO

\begin{tabular}{lrl}
\hline Enxerto & Veia safena & Art. mamária \\
\hline Pérvio sem lesão & $21(91,3 \%)$ & $3(100 \%)$ \\
Ocluído & $1(4,3 \%)$ & - \\
Pérvio com lesão $(\geqslant 50 \%)$ & $1 *(4,3 \%)$ & - \\
\hline Total & 23 & 3 \\
angioplastia na PS-CD $(90 \rightarrow 20 \%)$ & \\
\hline
\end{tabular}


DALLAN, L. A.; OLIVEIRA, S. A.; CECCHY, H.; AIRÊ, S.; SABINO NETO, A.; IGLÉZIAS, J. C. R.; VERGINELLI, G.; JATENE, A. D. - Revascularização miocárdica de urgência após complicação de angioplastia tranșluminal coronária: abordagem cirúrgica atual. Rev. Bras. Cir. Cardiovasc., 5(1): 42-53, 1990.

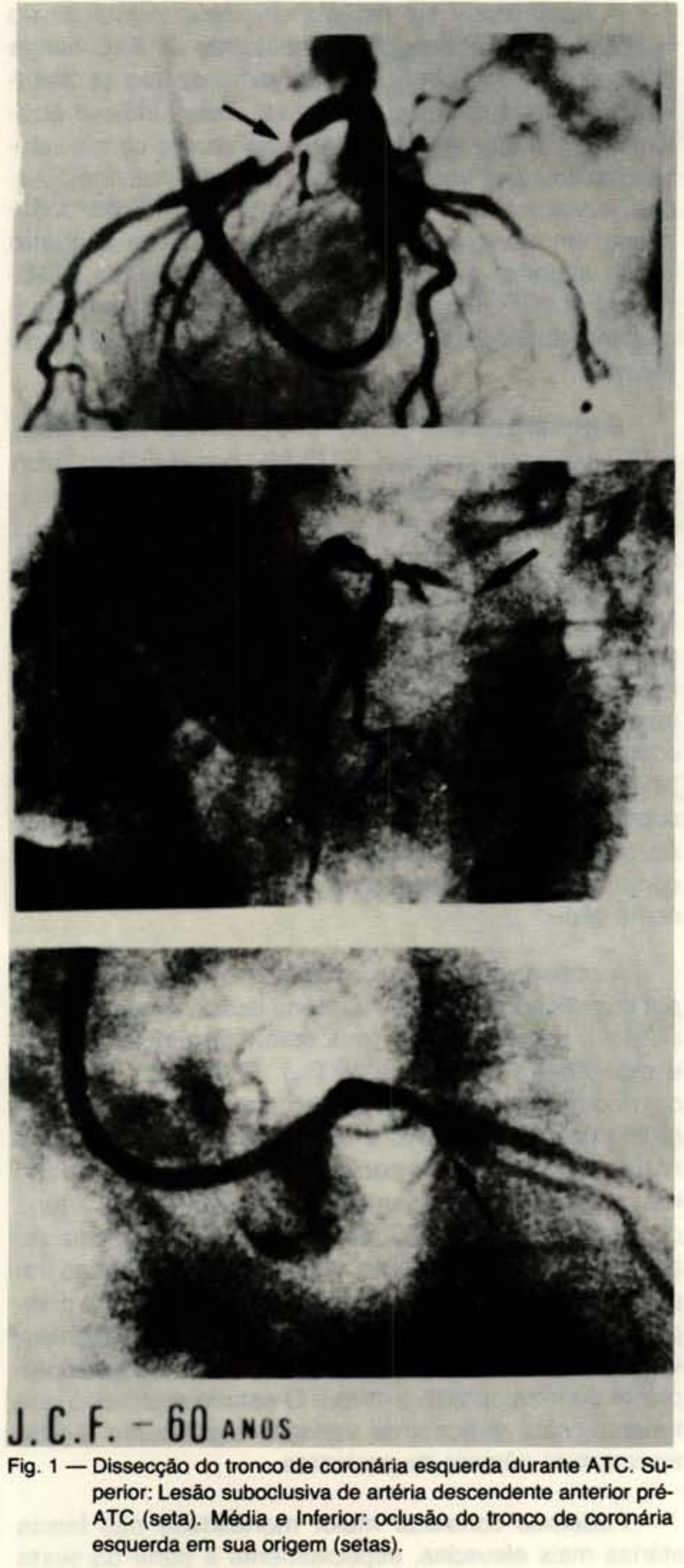

No Instituto do Coração, a ATC começou a ser realizada em 1981. A indicação, inicialmente restrita a pacientes com lesōes uniarteriais e proximais, foi rapidamente expandida para lesōes mais complexas, para síndromes isquêmicas agudas, assim como em pacientes previamente submetidos a cirurgia de revascularização do miocárdio. Hoje, a ATC também faz parte do arsenal utilizado

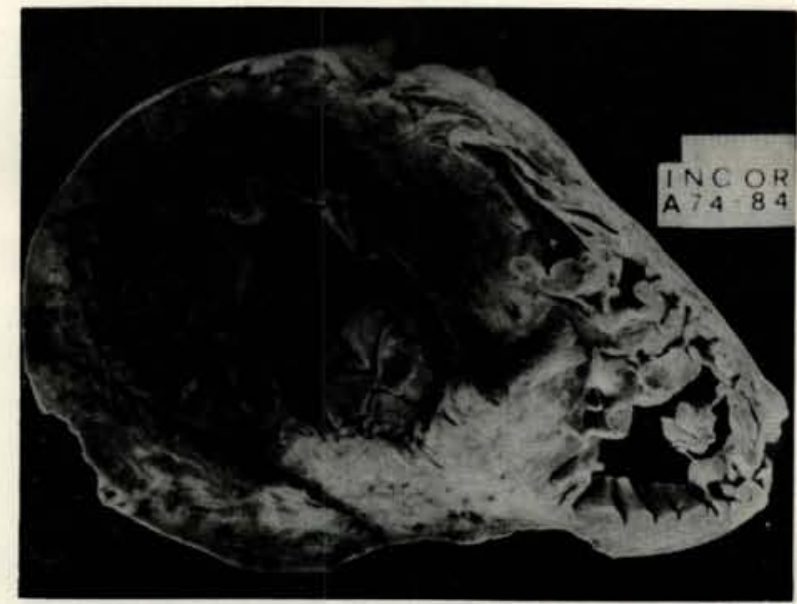

Fig. 2 - Corte transversal do coração da pacientes da Figura 1. Nota-se intenso infiltrado hemorrágico comprometendo todo o ventriculo esquerdo, decorrente da revascularização cirúrgica da área recém-infartada.

no tratamento de pacientes idosos e de outros com má funçāo ventricular ${ }^{25}$.

A maior limitação da ATC consiste na reestenose arterial coronária, que ocorre em aproximadamente $30 \%$ dos pacientes nos primeiros seis meses e em outros $15 \%$ entre dois e sete anos ${ }^{13},{ }^{18}$. Entretanto, apesar da progressiva experiência adquirida e do aprimoramento no material utilizado, o método não está isento de morbidade e mortalidade. A dilataçāo da artéria poderá não ser obtida, ou o procedimento poderá se complicar com a oclusão da artéria, em porcentagens variáveis de pacientes. Diferentes estudos mostram cifras que oscilam de $2 \%$ a $12,2 \%$ na incidência de oclusão, durante, ou logo após o procedimento $5,11,17,22,33$.

O desprendimento da placa aterosclerótica é o mecanismo mais freqüente de oclusão arterial pela ATC. Em $59(74,7 \%)$ de nossos pacientes, pudemos constatar sua ocorrência, com $4(6,8 \%)$ óbitos, o que coincide com os dados de literatura ${ }^{19,24}$. Entretanto, em $8(10,1 \%)$ pacientes, a dissecção da camada íntima arterial que se iniciou no local da angioplastia extendeu-se, retrogradamente, até o tronco da coronária esquerda, ocluindo-o (Figura 1). A isquemia miocárdica que se seguiu agravou severamente a função ventricular esquerda, culminando com o óbito de $50 \%$ deles (Tabela 3), (Figura 2).

A hemorragia subintimal, o espasmo coronário e a trombose local constituem outras causas de oclusão arterial pós ATC. A trombose coronária pode ocorrer de imediato, ou algumas horas após o procedimento ${ }^{39}$, período em que os pacientes já deixaram a sala de hemodinâmica. Esse fato pode acarretar perda de tempo entre a detecção da oclusão arterial e a efetiva reperfusão da área isquêmica, elevando a mortalidade. Em nossa casuística, $3(42,9 \%)$ dentre os sete pacientes desse grupo, vieram a falecer (Tabela 3 ). Recomendamos a 
DALlan, L. A., OliVeIRA, S. A.; CECCHY, H.; AIRÊ, S.; SABINO NETO, A.; IGLÉZIAS, J. C. R.; VERGINELLI, G.; JATENE, A. D. - Revascularização miocárdica de urgência após complicação de angioplastia transluminal coronária: abordagem cirúrgica atual. Rev. Bras. Cir. Cardiovasc., 5(1): 42-53, 1990.

vigilância próxima dos pacientes nas primeiras horas após ATC, se possível sob monitorização cardíaca em unidades especializadas.

A incidência de cirurgia de urgência pós ATC em diferentes centros de Cardiologia varia de $2 \%$ a $7 \%{ }^{4}$. 9, 16, 37 . Em nossa casuística, $3,2 \%$ de todos os pacientes submetidos a ATC necessitaram esse tipo de intervenção. Cumpre destacar que esse valor inclui, não só os pacientes com angina estável, mas também aqueles submetidos a ATC de urgência por angina instável, ou durante a fase aguda do infarto do miocárdio.

No início de nossa experiência, a cirurgia de revascularização do miocárdio era desencadeada de imediato, sempre que houvesse evidência de oclusão arterial durante a ATC. Nos últimos dois anos, entretanto, o emprego de cateteres de reperfusão preconizados por HINOHARA et alii ${ }^{15}$ tem permitido restabelecer rapidamente o fluxo sangüíneo através da artéria comprometida. Esse artifício possibilitou, em diversos pacientes, a regressão da isquemia miocárdica e a estabilização da área de dissecçãoo coronária, dispensando a intervenção cirúrgica de urgência. SIMPFENDORFER et alii ${ }^{35}$ relataram sucesso na redilataçăo de $27(87 \%)$, dentre 31 pacientes com oclusão arterial aguda por ATC, dos quais apenas $9(29 \%)$ necessitaram cirurgia de urgência.

No Instituto do Coração, o emprego recente na ATC de cateter guia com extremidade atraumática, associado, quando necessário, ao cateter de reperfusão, tem reduzido, significativamente, a necessidade de operaçōes de urgência ${ }^{2}$. No ano de 1987 , foram realizadas 670 ATC, das quais $13(1,9 \%)$ necessitaram cirurgia de urgência. Em 1988, esse número reduziu-se para $8(1,2 \%)$ dentre as 643 ATC realizadas. E nos últimos 14 meses, apenas $2(0,3 \%)$, dentre a 708 ATC, culminaram com cirurgia de urgência (Tabela 2). Por outro lado, a manutenção do fluxo anterógrado, mesmo diante da oclusão coronária, permite ao cirurgiāo atuar em condiçōes mais favoráveis, possibilitando a revascularização miocárdica mais completa, inclusive com o uso da artéria mamária interna.

A incidência de IAM após ATC mal sucedida é elevada. $\mathrm{Na}$ experiência de diversos autores, incluindo estudos multicêntricos, esta cifra é variável de $21 \%$ a $68 \%{ }^{3}$, 7. 20, 28, 36. As taxas mais altas coincidem com os pacientes que apresentaram instabilidade hemodinâmica durante ou logo após a ATC ${ }^{6}$. Observamos alteraçōes de ECG e/ou elevação significante de CKMB no pós-operatório de $32(40,5 \%)$ de nossos pacientes. Lembramos, entretanto, que esse valor inclui todos os pacientes operados, inclusive aqueles cuja indicaçăo da ATC deu-se por angina incontrolável ou IAM já instalado. Se considerarmos apenas os pacientes submetidos eletivamente a ATC, a taxa de IAM pós-operatório foi de $35,6 \%$.
A mortalidade em cirurgia de revascularizaçăo do miocárdio de urgência por complicaçăo de ATC atinge cifras significativamente mais elevadas do que as observadas na revascularizaçăo eletiva. Esses índices oscilam, na literatura, especialmente de acordo com a seleção prévia a que são submetidos os pacientes nos diversos Serviços. KILLEN et alii ${ }^{20}$ observam $11,3 \%$ de mortalidade em cirurgia de urgência pós ATC, taxa quatro vezes superior à revascularização programada. NAUNHEM et alii ${ }^{25}$ relataram $11 \%$ de mortalidade em situaçăo semelhante, contra apenas $1 \%$ na revascularização eletiva.

Em nossa casuística, de 1530 pacientes submetidos a ATC por angina estável, $59(3,8 \%)$ necessitaram cirurgia de urgência, ocorrendo $7(11,9 \%)$ óbitos. Essa mortalidade operatória também superou, várias vezes, nossos índices de mortalidade na revascularizaçāo eletiva do miocárdio. De 112 pacientes submetidos a ATC por angina instável (SI), $11(9,8 \%)$ necessitaram cirurgia de urgência, falecendo $2(18,2 \%)$ pacientes e, em 789 pacientes submetidos a ATC durante a fase aguda do infarto, apenas $9(1,1 \%)$ necessitaram operaçāo de urgência, ocorrendo $3(33,3 \%)$ óbitos (Tabela 1). Entretanto, entre janeiro de 1989 e fevereiro de 1990, 708 pacientes foram submetidos a ATC por diversas indicaçōes, dos quais apenas $2(0,3 \%)$ necessitaram cirurgia de urgência para correção de complicaçōes no procedimento, sem óbitos nesta série.

A maioria de nossas cirurgias de urgência deu-se por complicaçāo da ATC na artéria descendente anterior $(72,1 \%)$, seguida pela artéria coronária direita $(22,8 \%)$ e pela artéria circunflexa $(5,1 \%)$. Entretanto, a artéria cuja complicaçāo mais levou ao óbito foi a circunflexa ( $50 \%$ dos casos), devido a dissecção no tronco da coronária esquerda em dois pacientes desse grupo. Por outro lado, entre os 18 pacientes com obstruçảo da artéria coronária direita na ATC, somente $1(5,5 \%)$ faleceu. Ao analisá-los individualmente, verificamos que apenas um paciente apresentou baixo débito cardíaco após a complicação da ATC, e o bloqueio atrioventricular total observado em dois deles foi transitório e contornado pelo implante de marcapasso externo. O estudo estatístico, entretanto, não demonstrou variaçăo significante devido ao reduzido número de pacientes.

Pudemos constatar maior mortalidade nas faixas etárias mais elevadas, especialmente a partir da sexta década, como sugere a maioria dos trabalhos ${ }^{26}$. Entretanto, ao contrário de outros autores, não observamos correlação entre o número de artérias comprometidas por paciente $(\geqslant 70 \%)$ e a mortalidade.

O período decorrido entre a oclusão arterial na ATC e a efetiva revascularização do miocárdio guardou significado estatístico com os danos miocárdicos e a mortalidade (Tabela 4). Se, por um lado, medidas como a redilatação coronária ${ }^{19,35,36}$, o emprego de cateteres de reper- 
DALLAN, L. A.; OLIVEIRA, S. A.; CECCHY, H.; AIRÊ, S.; SABINO NETO, A.; IGLÉZIAS, J. C. R.; VERGINELLI, G.; JATENE, A. D. - Revascularização miocárdica de urgência após complicação de angioplastia transluminal coronária: abordagem cirúrgica atual. Rev. Bras. Cir. Cardiovasc., 5(1): 42-53, 1990.

fusăo ${ }^{12,}{ }^{16}$, ou, mesmo, a instalaçăo de endopróteses intracoronárias (stents) ${ }^{34}$ têm contribuido para restabelecer o fluxo coronário em grande número de pacientes, não podemos ignorar que, muitas vezes, o insucesso desses procedimentos pode adicionar minutos ou horas de isquemia miocárdica, comprometendo ainda mais os resultados cirúrgicos.

O baixo débito cardíaco que se segue à ATC mal sucedida está associado a maiores índices de mortalidade pós-operatória ${ }^{6}$. Dentre os 57 pacientes de nossa casuística que se encontravam em situaçāo hemodinâmica estável, apenas $3(5,2 \%)$ faleceram. Entretanto, dos 22 pacientes que necessitavam drogas inotrópicas ou suporte circulatório (balāo intra-aórtico) após a ATC, $9(40,9 \%)$ faleceram. Nove deles encontravam-se sob massagem cardíaca externa, dos quais apenas 4 $(44,4 \%)$ sobreviveram (Tabela 5 ).

Essa elevada mortalidade nos levou a procurar métodos de proteção miocárdica que permitissem baixar esses índices. Trabalhos recentes sugerem que a reperfusăo sangüínea sob pressão elevada em miocárdio submetidos a anóxia prolongada pode resultar em agressão adicional ${ }^{10}$ e que a alteração na composição das soluçōes de reperfusão pode ser benéfica ${ }^{1,21,23,31}$.

Nos últimos cinco pacientes, empregamos a técnica proposta por PARTINGTON et alii ${ }^{27}$, que utilizam cardioplgia sangüinea com induçăo e reperfusăo normotér- micas, administrada por via anterógrada (raiz da aorta) e retrógrada (seio coronário). Nāo observamos mortalidade ou complicações pós-operatórias entre esses pacientes, apesar de três deles necessitarem suporte inotrópico antes da revascularização do miocárdio. O número pequeno de casos não possibilitou conclusōes definitivas. Entretanto, o método parece promissor na redução da mortalidade e na prevençăo das complicaçōes mais freqüentemente observadas no pós-operatório desses pacientes.

O baixo débito cardíaco pós-operatório foi a causa mais comum de óbito hospitalar em nossa série $(66,7 \%)$. Outros $4(33,3 \%)$ pacientes faleceram por motivos diversos, especialmente infecçăo (Tabela 6).

Dentre os 54 pacientes com seguimento tardio, 43 $(79,6 \%)$ encontram-se assintomáticos e 5 (9,3\%) em classes funcionais II e III. Foram observados quatro óbitos nos 103 meses de seguimento, dos quais apenas dois de causa cardíaca.

Tudo sugere que a ATC continuará a ser empregada extensivamente. As alteraçōes introduzidas na técnica reduziram significativamente a necessidade de cirurgia de urgência. Paralelamente, as modificaçōes no procedimento cirúrgico, especialmente na proteçāo miocárdica, foram benéficas. Um perfeito entendimento entre as equipes de hemodinâmica e cirurgia certamente é necessário para a obtençăo de bons resultados.

RBCCV 44205-106

DALLAN, L. A.; OLIVEIRA, S. A.; CECCHY, H.; AIRÊ, S.; SABINO NETO, A.; IGLÉZIAS, J. C. R.; VERGINELLI, G.; JATENE, A. D. - Emergency myocardial revascularization after complicated percutaneous transluminal coronary angioplasty: actual surgical approach. Rev. Bras. Cir. Cardiovasc., 5(1): 42-53, 1990.

ABSTRACT: Between July 1981 and February 1990, 2431 patients underwent attempted percutaneous transluminal coronary angioplasty (PTCA). Seventy-nine (3.2\%) patients subsequently underwent emergency miocardial revascularization, and $32(40.5 \%)$ developed myocardial infarction with $12(15.2 \%)$ deaths. The operative mortality rate was significantly increased among elderly patients, those with left main occlusive dissection, late coronary thrombosis, and specially among patients with hemodynamic instability after PTCA. Additional minutes or hours of low myocardial and systemic perfusion after failed PTCA also increase the risk of immediate surgical revascularization. Despite the growing role of PTCA in the treatment of more complex coronary atherosclerotic heart diseases, the number of failed procedures that necessitate coronary artery bypass surgery decreased last two years (10/1351). This have been possible by placing a reperfusion catheter (Stack) across the narrowed coronary artery lumen. Surgical results were consistently improved by modifications in myocardial protection techniques, besides the use of reperfusion catheter after failed PTCA.

DESCRIPTORS: myocardial revascularization, angioplasty; myocardial revascularization, surgery; myocardial protection, cardioplegia. 
DALLAN, L. A.; OLIVEIRA, S. A.; CECCHY, H.; AIRÊ, S.; SABINO NETO, A.; IGLÉZIAS, J. C. R.; VERGINELLI, G.; JATENE, A. D. - Revascularização miocárdica de urgência após complicaçāo de angioplastia transluminal coronária: abordagem cirúrgica atual. Rev. Bras. Cir. Cardiovasc., 5(1): 42-53, 1990.

\section{REFERÊNCIAS BIBLIOGRÁFICAS}

1 ALLEN, B. S.; OKAMOTO, F.; BUCKBERG, G. D.; BUGY, H.; YOUNG, H.; LEAF, J.; BEYERSDORF, F.; SJOSTRAND, F.; MALONEY Jr., J. V. - Immediate functional recovery after six hours of regional ischemia by careful control of conditions of reperfusion and composition of reperfusate. J. Thorac. Cardiovasc. Surg., 92: 621-635, 1986 .

2 ARIÊ, S.; CHECCHI, H.; RATI, M. A. N.; BELLOTTI, G. M. V.; PILEGGI, F. J. C. - Angioplastia coronária: estudo atual e perspectivas. Rev. Assoc. Med. Brasil., 35: 151-158, 1989.

3 ARNONI, A. S.; SOUZA, L. C. B.; FRANCISCO, R. M. G. C.; SOUZA, G. C.; KANTROWITZ, L.; OLIVEIRA, J. B.; DINKHUYSEN, J. J.; MORAES, A. G.; SOUSA, J. E. M. R.; JATENE, A. D.; PAULISTA, P.P. - Cirurgia de emergência após angioplastia transluminal coronária. Arq. Bras. Cardiol., 46: 311-315, 1986.

4 BLOCK, P. C. - Percutaneous transluminal coronary angioplasty: role in the treatment of coronary artery disease. Circulation, 72 (Supl. 5): 161-165, 1985.

5 BREDLAU, E. C.; ROUBIN, G. S; LEIMGRUBER, P. P.; DOUGLAS Jr., J. S.; KING III, S. B.; GRUENTZIG, A. R. - In hospital morbidity and mortality in patients undergoing elective coronary angioplasty. Circulation, 72: 1044-1052, 1985.

6 CONNOR, A. R.; VLIETSTRA, R. E.; SCHAFF, H. V.; ILSTRUP, D. M.; ORSZULAK, T. A. - Early and late results of coronary artery bypass after failed angioplasty. J. Thorac. Cardiovasc. Surg., 96: 191-197, 1988.

7 COWLEY, M. J.; DORROS, G.; KELSEY, S. F.; VAN RADEN, M.; DETRE, K. M. - Acute coronary events associated with percutaneous transluminal coronary angioplasty. Am. J. Cardiol., 53: 12C-16C, 1984.

8 COWLEY, M. J.; DORROS, G.; KELSEY, S. F.; VAN RADEN, M.; DETRE, K. M. - Emergency coronary bypass surgery after coronary angioplasty: The National Heart Lung and Blood Intitute's percutaneous transluminal coronary angioplasty registry experience. Am. J. Cardiol., 53: 22C-26C, 1984.

9 COWLEY, M. J.; VETROVEC, G. W.; DISCIASCIO, G.; LEWIS, S. A.; HIRSH, P. D.; WOLFGANG, T. C. Coronary angioplasty of multiple vessels: short-term outcome and long-term results. Circulation, 6: 1314-1320, 1985.

10 DALLAN, L. A. O.; OLIVEIRA, S. A.; LAVITOLA, P.; VERGINELLI, G.; JATENE, A. D. - Infarto hemorrágico conseqüente à reperfusão miocárdica: relato de quatro casos. Arq. Bras. Cardiol., 54: 127-132, 1990.

11 ELLIS, S.; ROUBIN, G.; GOX, W. - Angiographic and clinical prediction of acute closure after coronary angioplasty. Circulation, 74 (Supl. 2): 194, 1986. (Resumo).
12 FERGUSON, T. B.; HINOHARA, T.; SIMPSON, J.; STACK, R. S.; WECHSLER, A. S. - Catheter repertusion to allow optimal coronary bypass grafting following failed transluminal coronary angioplasty. Ann. Thorac. Surg., 42: 399-405, 1986.

13 GRUENTZIG, A. R.; KING, S. B.; SCHLUMPF, M.; SIEGENTHALER, W. - Long-term follow-up after percutaneous trans-luminal coronary angioplasty: the early $\mathrm{Zu}$ rich experience. $\quad$ N. Engl. J. Med., 316: 1127-1132, 1987.

14 GRUENTZIG, A. R.; MYLER, R. K.; HANNA, E. S.; TURINA, M. I. - Transluminal angioplasty of coronary artery stenosis. Circulation, 56 (Supl. 2): 84-90, 1977.

15 HINOHARA, T.; SIMPSON, J. B.; PHILLIPS, H. R.; BEHAR, V. S.; PETER, R. H.; KONG, Y.; CARLSON, E. B.; STACK, R. S. - Transluminal catheter reperfusion: a new technique to reestablish blood flow after coronary occlusion during percutaneous transluminal coronary angioplasty. Am. J. Cardiol., 1: 684-686, 1986.

16 HINOHARA, T.; SIMPSON, J. B.; PHILLIPS, H. R.; STACK, R. S. - Transluminal intracoronary reperfusion catheter: a device to maintain coronary perfusion between failed coronary angioplasty and emergency coronary bypass surgery. J. Am. College Cardiol., 5: 977-982, 1988.

17 HOLLMAN, J.; CRUENTZIG, A. R.; DOUGLAS, J. S.; KING, S. B.; ISCHINGER, T.; MEIER, B.; Acute occlusion after percutaneous transluminal coronary angioplasty: a new approach. Circulation, 68: 725-732, 1983.

18 HOLMES Jr., D. R.; VLIESTRA, R. E.; SMITH, H. C. Reestenosis after percutaneous transluminal coronary angioplasty (PTCA): a report from PTCA registry of the National Heart, Lung and Blood Institute. Am. J. Cardiol., 53: 77C-81C, 1984.

19 ISCHINGER, T.; ZACK, P.; AKER, U. - Acute coronary occlusion during balloon angioplasty due to intracoronary thrombus and coronary spasm: a reversible complication. Am. Heart J., 107: 1271-1275, 1984.

20 KILLEN, D. A.; HAMAKER, W. R.; REED, W. A. - Coronary artery bypass following percutaneous transluminal coronary angioplasty. Ann. Thorac. Surg., 40: 133-138, 1985.

21 KLONER, R. A.; ELLIS, S. G.; LANGE, R.; BRAUNWALD, E. - Studies of experimental coronary artery reperfusion: effects on infarct size, myocardial function, biochemistry, ultrastructure and microvascular damage. Circulation, 68 (Supl. 1): 8-15, 1983.

22 MARQUIS, J. F.; SCHWARTZ, J. F.; SCHWARTZ, L.; ALDRIDGE, H.; MAJID, P.; HENDERSON, M.; MATUSHINSKY, E. - Acute coronary artery occlusion during percutaneous transluminal coronary angioplasty treated by redilation of the occluded segment. J. Am. College Cardiol.,4: 1268-1271, 1984. 
DALLAN, L. A.; OliveIRA, S. A.; CECCHY, H.; AIRÊ, S.; SABINO NETO, A.; IGLÉZIAS, J. C. R.; VERGINELLI, G.; JATENE, A. D. - Revascularização miocárdica de urgência após complicaçăo de angioplastia transluminal coronária: abordagem cirúrgica atual. Rev. Bras. Cir. Cardiovasc., 5(1): 42-53, 1990.

23 METZDORFF, M. T.; GRUNKEMEIER, G. L.; STARR, A. - Effect of initial reperfusion temperature on myocardial preservation. J. Thorac. Cardiovasc. Surg., 91: 535-550, 1986.

24 MURPHY, D. A.; CRAVER, J. M.; JONES, E. L.; GRUENTZIG, A. R.; KING III, S. B.; HATCHER, C. R. - Surgical revascularization following unsuccessful percutaneous transluminal coronary angioplasty. J. Thorac. Cardiovasc. Surg., 84: 342-348, 1982.

NAUNHEIM, K. S.; FIORE, A. C.; FAGAN, D. C.; McBRIDE, L. R.; BARNER, H. B.; PENNINGTON, G.; WILLMAN, V. L.; KERN, M. J.; DELIGONUL U.; VANDORMAEL, M. C.; KAISER, G. C. - Emergence coronary artery bypass grafting for failed angioplasty: risk factors and outcome. Ann. Thorac. Surg., 47: 816-823, 1989.

26 PARSONNET, V.; FISCH, D.; GIELCHINSKY, I. - Emergency operation after failed angioplasty. J. Thorac. Cardiovasc. Surg., 96: 198-203, 1988.

27 PARTINGTON, M. T.; ACAR, C. R.; JULIA, P. L.; BUCKBERG, G. D. - Anterograde/retrograde blood cardioplegia: a combined tecnique allowing global myocardial protection in jeopardized myocardium. Am. Heart Assoc. Circ. Suppl., 76: 656-660, 1987.

28 PELletieR, L. C.; PARDINI, A.; RENKIN, J.; DAVID, P. R.; HEBERT, Y.; BOURASSA, M. G. - Myocardial revascularization after failure of percutaneous transluminal coronary angioplasty. Thorac. Cardiovasc. Surg., 90: 265-271, 1985.

29 ROBERTSON, J. M.; VINTEN-JOHANSEN, J.; BUCKBERG, G. D.; ROSENKRANZ, E. R.; MALONEY Jr., J. - Safety of prolonged aortic clamping with blood cardioplegia: I. Guatamate enrichment in normal hearts. J. Thorac. Cardiovasc., Surg., 88: 395-401, 1984.

30 ROSENKRANTZ, E. R.; BUCKBERG, G. D.; LAKS, H.; MULDER, D. G. - Warm induction of cardioplegia with glutamate-enriched blood in coronary patients with cardiogenic shock who are dependent on inotropic drugs and intra-aortic balloon support. J. Thorac. Cardiovasc. Surg., 86: 507-518, 1983.

31 ROSENKRANTZ, E. R.; OKAMOTO, F.; BUCKBERG, G. D.; ROBERTSON, J. M.; VINTEN-JOHANSEN, J.; BUGYI, H. I. - Safety of prolonged aortic clamping with blood cardioplegia: III. Aspartate enrichment of glutamate blood cardioplegia in energy depleted hearts after ischemic and repertusion injury. J. Thorac. Cardiovasc. Surg., 91: 428-435, 1986.

ROSENKRANTZ, E. R.; OKAMOTO, F.; BUCKBERG, G. D.; VINTEN-JONHANSEN, J.; ROBERTSON, J. M.; BUGYIH, H. - Safety of prolonged aortic clamping with blood cardioplegia: II. Glutamate enrichment in energy-depleted hearts. J. Thorac. Cardiovasc. Surg., 88: 402-410, 1984.
33 SHIU, M. F.; SILVERTON, N. P.; OAKLEY, D.; CUMBERLAND, D. - Acute coronary occlusion during percutaneous transluminal coronary angioplasty. Br. Heart J., 54: 129-133, 1985.

34 SIGWART, U.; PUEL, J.; MIRKOVITCH, V.; JOFFRE, F.; KAPPENBERGER, L. - Intravascular stents to prevent occlusion and reestenosis after transluminal angioplasty. New Engl. J. Med., 316: 701-706, 1987.

35 SIMPFENDORFER, C.; BELARDI, J.; BELLAMY, G.; GALAN, K.; FRANCO, I.; HOLLMAN, J. - Frequency, management and follow-up of patients with coronary occlusions after percutaneous transluminal coronary angioplasty. $\quad A m$. J. Cardiol., 59: 267-269, 1987.

36 SINCLAIR, N.; FRACP, M. G.; MCCABE, H. C.; SIPPERLY, M. E.; BAIM, D. S. - Predictors, therapeutic options and long-term outcome of abrupt reclosure. Am. J. Cardiol., 61: 61G-66G, 1988.

37 STARK, K. S.; SATLER, L. F.; KRUCOFF, M. W.; RACKLEY, C. E.; KENT, K. M. - Myocardial salvage after failed coronary angioplasty. J.Am. College Cardiol., 15: 78-82, 1990.

38 TALLEY, J. D.; JONES, E. L.; WEINTRAUB, W. B.; KING III, S. B. - Coronary artery bypass surgery after failed elective percutaneous transluminal coronary angioplasty. Circulation, 79 (Supl. 1): 126-131, 1989.

39 TREMBLAY, G.; PARRIS, T. M.; MINTZ, G. S. - Acute occlusion during coronary angioplasty. J. Invasive Cardiol, 1: 55-57, 1988.

\section{Discussão}

\section{DR. JOSE LUIS FILGUEIRA Montevideu, Uruguai}

Felicitamos a los autores por su extensa experiencia en el tema, los buenos resultados obtenidos, en especial con las modernas técnicas de protección miocárdica preconizadas por Buckberg. Sus resultados coinciden con los de la literatura en el sentido de que la mortalidad quirúrgica es mayor en aquellos pacientes que previamente fueron sometidos a A.T.C. por infarto de miocardio $(33,3 \%)$ que los que representaban angor inestable $(18,18 \%)$ y los que se les efectuaba una A.T.C. electiva $(11,86 \%)$. También es coincidente el hecho de que los pacientes operados en condiciones hemodinámicas estables tienen menor mortalidad y menos complicaciones post operatorias (síndrome de gasto bajo, infarto agudo de miocardio y aún sangrado post operatorio) que los operados con síndrome de gasto bajo y más aún si necesitaron maniobras de reanimación ( $J$. Thorac. Cardiovasc. Surg., 96: 198-203, 1988). Un dato que tiene valor pronóstico es si luego de A.T.C. queda o no una oclusión completa del vaso tratado. De ahí surgirá la utilización de catéteres de reperfusión con múltiples orificios latera- 
DALLAN, L. A.; OlIVEIRA, S. A.; CECCHY, H.; AIRÊ, S.; SABINO NETO, A.; IGLÉZIAS, J. C. R.; VERGINELLI, G.; JATENE, A. D. - Revascularização miocárdica de urgência após complicação de angioplastia transluminal coronária: abordagem cirúrgica atual. Rev. Bras. Cir. Cardiovasc., 5(1): 42-53, 1990.

les que permiten mantener el flujo sanguíneo a través de la lesión y así mejorar las condiciones hemodinámicas en que se lleva al paciente a sala de operaciones. EI rol del equipo quirúrgico es muy importante en el paciente que se va a realizar un A.T.C., viendo al enfermo previamente y discutiendo con el hemodinamista las situaciones límites; por ejemplo, pacientes que previamente habian sido sometidos a cirugía de revascularización miocárdica y luego se va a realizar un A.T.C.: si se complica y hay que re-operar-lo, el acceso al corazón no puede ser tan rápido. En estos casos hay que ver previamente: protocolo operatorio (se cerró pericardio?), RX, tórax (proximidad de V.D. al corazón), palpar ambos pulsos femorales pensando que se debe colocar balón de contrapulsación intra-aórtico a la menor duda y algunos centros preconizan la aplicación de by-pass cardiopulmonar percutáneo (Ann. Thorac. Surg., 47: 121, 1989). Coincidimos que en estas situaciones se deben tomar al máximo las medidas de protección miocárdica en su más amplia acepción y dentro de ellas toma relevancia la técnica de infusión cardiopléjica retrógrada para poder alcanzar el área miocárdica comprometida por la oclusión coronaria. No ha resultado útil, cuando existe disección de la arteria coronaria, efectuar la arteriotomía durante la inyección de cardioplejia retrógrada. Creemos que situaciones especiales: pacientes jóvenes, buena función ventricular izquierda, compromiso de arteria descendente anterior bien desarrollada y especialmente si presenta una condición hemodinámica estable, se puede utilizar la arteria mamaria interna. No nos debemos olvidar que el factor predictivo más importate en el pronóstico alejado es si se empleó o no la arteria mamaria interna en el procedimiento de revascularización.

\section{DR. FEDERICO BENETTI}

\section{Buenos Aires, Argentina}

Gostaria de felicitar o Dr. Dallan e seu grupo de trabalho, pela apresentação desta série muito grande de pacientes e, especialmente, pelos resultados excelentes. Calculo que sobre 79 pacientes operados de urgência, nove foram enviados à sala de cirurgia sob massagem cardíaca e a mortalidade global foi de apenas $15 \%$, o que é, realmente, excelente. O número de pacientes em que se empregou a técnica de Buckberg foi pequeno, porém a mesma parece ser promissora. Eu, entretanto, nāo tenho nenhuma experiência com a técnica de Buckberg, nesse tipo de paciente, e vou seguir observando atentamente os vossos resultados. No ano passado, nós operamos de urgência cinco pacientes, nos quais houve fracasso da angioplastia coronária e em quatro não empregamos a circulação extracorpórea. Não houve óbitos. Uma senhora, que teve dissecção do tronco da coronária esquerda, foi operada com circulação extracorpórea e veio a falecer. Duas dessas pacientes do ano passado apresentavam lesão isolada na artéria descen- dente anterior e a artéria coronária anteriormente e posteriormente à placa era normal. Isso me chamou muito a atenção, porque, nesses tipos de pacientes, nāo indicamos a angioplastia coronária e, sim, diretamente a revascularização do miocárdio sem circulação extracorpórea. Voltando à análise do número total de pacientes, acho interessante ressaltar que a maioria tinha angina estável, lesão coronária uniarterial, estava numa faixa etária elevada, recebeu em média 1,57 pontes e a mortalidade extrapolada foi de $0,5 \%$. Essa mortalidade é a que nós obtemos hoje nesse tipo de paciente com cirurgia sem circulação extracorpórea. Ou seja, estamos diante de um procedimento cuja mortalidade primária é tão elevada quanto a cirurgia. Eu creio que esses pacientes com lesão da descendente anterior devam ser mais exaustivamente investigados e devemos propor estudos randomizados para sabermos o que acontecerá a curto e a longo prazo. Sou da opinião de que, sobretudo em faixas etárias mais altas, eles devam ser tratados pela cirurgia como primeiro procedimento. Recentemente, surgiu, na literatura, um trabalho que compara esses dois grupos de pacientes, demonstrando que a sobrevida com a cirurgia é maior. Creio que devemos enfatizar a realização desse tipo de estudo. Para finalizar, quero felicitar o Dr. Dallan, novamente,, por seu trabalho e deixar uma pergunta: por que nós, cirurgióes, temos que assumir o risco de operar pacientes com mortalidade 20 ou 30 vezes maior do que se fossem operados primariamente? Obrigado.

\section{DR. DALLAN \\ (Encerrando)}

Gostaria de agradecer os comentários elogiosos do Dr. Federico Benetti, de Buenos Aires, e do Dr. Jose Luis Filgueira, de Montevideo. O Dr. Benetti posicionouse favoravelmente ao tratamento cirúrgico convencional, ao invés da angioplastia (ATC), em pacientes com lesão coronária uniarterial. Realmente, existe uma polêmica muito grande na indicaçăo angioplastia versus cirurgia, nesse tipo de paciente. Os excelentes resultados cirúrgicos obtidos, nāo só em nosso Serviço, mas também descritos na literatura, contribuem para essa polêmica. Não vou entrar, neste momento, no mérito da indicação de angioplastia, uma vez que não constitui objetivo deste trabalho e cuja controvérsia poderia, até, ser motivo de mesa-redonda composta por hemodinamicistas, clínicos e cirurgiōes. Entretanto, concordo com o Dr. Benetti, em que a cirurgia eletiva, para esses pacientes, tem mortalidade próxima de zero, e que, paradoxalmente, cada vez menos temos oportunidade de operar pacientes com lesão coronária uniarterial. Nesse sentido, vem sendo realizado, há aproximadamente dois anos, no Instituto do coração, um estudo prospectivo, randomizado, coordenado pelo Prof. Whady Hueb, que sorteia, alheiatoriamente, pacientes com lesão uniarterial para tratamento 
DALLAN, L. A.; OliveIRA, S. A.; CECCHY, H.; AIRÊ, S.; SABINO NETO, A.; IGLÉZIAS, J. C. R.; VERGINELLI, G.; JATENE, A. D. - Revascularizaçáo miocárdica de urgência após complicação de angioplastia transluminal coronária: abordagem cirúrgica atual. Rev. Bras. Cir. Cardiovasc., 5(1): 42-53, 1990.

cirúrgico convencional, angioplastia, ou tratamento clíni$c o$. Acredito que, num futuro muito próximo, já teremos resultados concretos desse estudo. Temos, entretanto, que partir da premissa de que a angioplastia é uma realidade. Calcula-se que, em 1990, devam ser realizadas mais de $\mathbf{4 0 0 . 0 0 0}$ angioplastias, apenas nos Estados Unidos. Temos, ainda, que lembrar que a indicação da angioplastia tem-se expandindo. Inicialmente limitada apenas para pacientes uniarteriais, com lesāo localizada e proximal, hoje vem sendo indicada para pacientes idosos, reoperaçōes e outros pacientes graves, especialmente com problemas pulmonares, o que nos obriga, periodicamente, a enfrentar esse tipo de complicação da ATC. Eu gostaria de ressaltar que a mudança radical na abordagem desses pacientes com complicação da ATC vem contribuindo, decisivamente, para melhorar seus resultados. Isso se deve em parte ao emprego de cateteres tipo Stack, que mantêm o fluxo através do local comprometido da artéria, possibilitando a estabilização da placa após sua dissecção, ou permitindo que o paciente seja operado em condiçốes hemodinâmicas favoráveis. O uso de cardioplegia sangüínea, por via anterógrada e retrógrada, com indução e reperfusão normotérmicas, como também proposto pelo Dr. Filgueira, vem, igualmente, propiciando um melhor desempenho cardiaco pós-operatório. Respondendo à pergunta formulada pelo Dr. Jazbik, informo que a incidência de infarto per-operatório, dentre os 77 pacientes operados de urgência, foi de $40,5 \%$. Quando nos reportamos apenas aos 59 pacientes submetidos, eletivamente, a ATC, esse índice caiu para $35,6 \%$, o que ainda consideramos uma cifra muito elevada, dada a boa condição ventricular pré-operatória da maioria desses pacientes. Em resposta ao Dr. Filgueira, informa que o Instituto do Coração também estabeleceu normas rígidas que são aplicadas a todos os pacientes submetidos a ATC. O paciente é preparado psicológica e fisicamente. Como rotina, são realizadas tricotomia e tipagem sangüínea prévias. Existe sala cirúrgica montada, com equipe médica e paramédica à disposição, para uma eventual cirurgia de emergência. Todo esse aparato tem abreviado o tempo real da revascularização cirúrgica desses pacientes e os resultados desse sincronismo refletiram-se, significativamente, na diminuiçăo da mortalidade intra e pós-operatória, como foi detaIhadamente mostrado durante a apresentação deste trabalho. Finalizo reiterando os meus agradecimentos aos comentaristas e à comissão organizadora deste congresso, por mais esta oportunidade. 